\title{
Application of iot technology in Teaching of electronic information system practice
}

\author{
Qing wan ${ }^{1, \text { a }}$ and Jianghua Liu ${ }^{2, b}$ \\ ${ }^{1}$ School of Electronics and Information Engineering, \\ Hubei University of Science and Technology Xiannning, China \\ ${ }^{2}$ School of Electronics and Information Engineering, \\ Hubei University of Science and Technology Xiannning, China \\ awanqin_002@126.com, bljhmail1979@126.com
}

Keywords: internet of thing, teaching reform, electronic information system.

\begin{abstract}
In this paper,we discuss the application of Internet of things technology in the teaching reform of electronic information system.The Internet of things technology is added to the e-information system practice curriculum.The project is driven by the Internet of things, leading students to study autonomously and independently design. Through recent years teaching practice proves that the introduction of the Internet of things technology practice curriculum reform is very effective on electronic information system, improve the students' exploring ability, innovation ability and practice ability.
\end{abstract}

\section{Introduction}

In recent years, iot technology has become a more and more popular research direction.Iot technology is essentially a combination of virtual digital technology and physical world.IoT technology is the core technology of realizing social informatization and intelligence. Its English name is "Internet of things". As the name implies, the Internet of things is the interconnection between devices and devices.At present, the Internet of things is the mobile Internet and other ways to connect and control more electrical equipment to make our life more convenient.Therefore, the concept of Internet of things is generated in this environment, which is the development direction of electronic technology in the future[1].

In the era of the Internet of things, all sorts of everydayobjects will have tinychips placed inside them and gain the ability toprocess information andtalk to the Web. The core of Internet of things technology is the construction of sensor network, which consists of processor, sensor and wireless communication module.The rise of low power network technology. For example, use the cc2530 wireless zigbee microcomputer with MCS51 as the core to realize the zigbee network, or to implement the ble mesh network based on the nrf51822 wireless ble microcontroller based on ARM.These devices can be used as the basic platform for the content of the Internet of things.

\section{Teaching reform of electronic information system practice curriculum}

2.1 Current Situation of Programming Teaching for electronic information system practice

The purpose of the electronic information system practice course is to improve students' ability in practice.It enables students to apply the techniques of electronic technology and single-chip microcomputer to engineering applications[2].The traditional e-information system practice course is based on the courses of analog electronic technology, digital electronic technology and SCM, and presents the topic of design content, as shown in table 1 below: 
Table 1. The topic of design.

\begin{tabular}{cc}
\hline The project name & The difficulty coefficient \\
\hline Design of digital voltmeter & height \\
Real-time clock design & low \\
Design of integrated dc stabilized voltage source & medium \\
Design of function signal generator & medium \\
Design of control circuit of rear lamp of automobile & height \\
Basketball 30S timing circuit design & medium \\
\hline
\end{tabular}

The practice of electronic information system is not focused on teaching, and students are divided into groups of three to four. Each group of students assigned work tasks and finally coordinated the design of the final work[3][4].This can actively mobilize the team spirit of the students, and lay the foundation for the teamwork of design projects in the post-graduation work.

The design requires that students first simulate the software through protues software, then complete the drawing of the hardware circuit diagram and make PCB.The students independently welded the design products, and finally carried out the comprehensive debugging of the system. The traditional project content is based on some functional basic design, and most of the projects have been proposed for a long time, which does not involve the newly emerging technology of things.Traditional projects lack integration with the latest technology and lack of innovation.

Electronic information system from the current practice of the course design of the teaching effect of point of view: with the new technology rapid development, especially in recent years, the high-speed development of Internet technology, makes the application of the current course training results have far cannot satisfy the requirement of society on college students' application ability.In order to adapt to the current market demand for college graduates, it is necessary and urgent to add new technologies, especially Internet of things technology to practical training practices.

\subsection{Application of Internet of things technology to teaching reform}

To reform the original teaching method, the knowledge of adding some Internet of things is to teach, and combined with the use of singlechip, some practical applications of the Internet of things are proposed.The main teaching objectives are guided teaching[5], which mainly involves several knowledge points of Internet of things: zigbee, bluetooth, GPS, etc.

The traditional teaching method is the teacher proposes the design topic.In this way, the design topic is ready-made, and the topic lacks innovation, so students can't contact the latest technology and adapt to the latest market demand. At the same time, fixed design topic can not mobilize students' autonomy and enthusiasm.In order to solve the above disadvantages, simultaneously explore students' creativity.A pair of teaching methods are proposed in practice teaching.The so-called one-to-many teaching method is the teacher puts forward the technical source, the many students compose the team to carry on the discussion. The specific implementation plan is shown in figure 1 below:

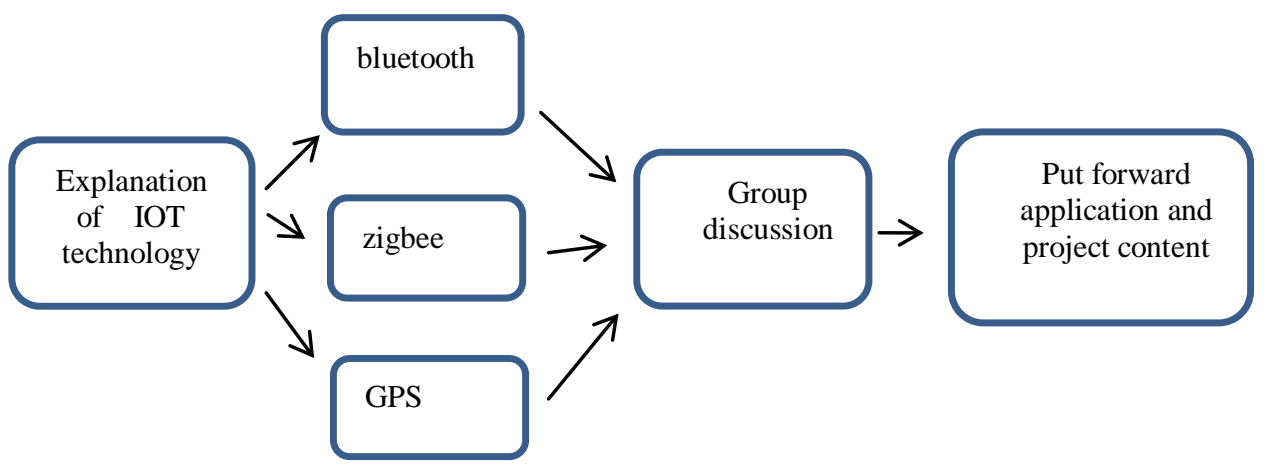

Fig.1. Design project proposal 
To learn more about these points, students need to spend more time collecting them through various means, such as the Internet and books.After laying the foundation, we will divide the students into groups to discuss, and leave a lot of time for students to explore and collaborate with each other.Through such a process, students can find the application situation and the basic direction of the design.In practice teaching, students proposed multiple project scheme. As shown in table 2 below:

Table 2. The topic of design.

\begin{tabular}{cc}
\hline The project name & The difficulty coefficient \\
\hline Wireless ordering system & height \\
GPS navigation system & medium \\
Smart home system & height \\
Building temperature control system & medium \\
\hline
\end{tabular}

After these design proposals, how do students finish the whole design process?The teaching of this process should be the teacher as the auxiliary function, the student as the main body. As a navigator and facilitator, teachers provide guidance in student design process to enhance students' interaction with teachers. The main body is a group of students, which is quite similar to a design team and needs to propose a design plan.Then we discuss the details of the design plan, such as hardware selection, principle feasibility analysis, cost estimation, etc.After doing this work, we can build a framework of the whole system and put forward the basic structure of the whole system.

The design of hardware and software can be started after the proposal is proposed.The hardware design is designed according to the previous system framework to design the circuit diagram. At the same time we draw software system block diagram, write system software.Before making PCB or hardware welding, we can use computer software to carry out the simulation, then we can start assembly and welding, and complete the design report after debug.The whole project completion process is shown in figure 2.

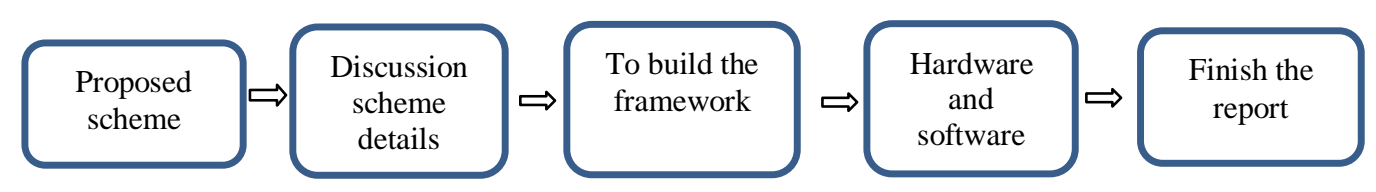

Fig.2. Design project completion

\section{Check and Assessment Phase}

The rational evaluation mechanism is the driving force of students' independent innovation. The Internet of things is the new technology and new content.Students need to do pre-study and data search before designing. Only by doing this can students better realize the design of classroom projects.After joining the Internet of things technology, the design project is an open project, which requires students to design the topic under the guidance of teachers.It is also important to ensure that they are not too difficult to implement or too simple.After designing the project, students begin to assemble and build, complete the design products, and finally accept the acceptance, which is the core of the course design.After the whole design process is completed, students need to summarize in writing and complete the experiment report.In order to achieve students' exchange experience, and to exercise students' expressive ability, it is necessary to make a speech defense of the whole project, which is also a very important part. For these links, we achieve the quantitative score. Each step is graded according to a certain percentage, as shown in table 3: 
Table 3. Three Scheme comparing.

\begin{tabular}{ccc}
\hline Step & proportion & fractions \\
\hline Pre-class preview and & $10 \%$ & 10 \\
information search & $20 \%$ & 20 \\
The project design & $30 \%$ & 30 \\
Product acceptance & $20 \%$ & 20 \\
Project report & $20 \%$ & 20 \\
Speech plea & $100 \%$ & 100 \\
The total & &
\end{tabular}

\section{Summary of teaching effect}

The first is to improve students' enthusiasm and autonomy, which greatly improves the passing rate of design courses.Traditional design projects have ready-made reference examples, which do not require students to explore and think themselves.Students only need to imitate the example to complete, do not need their own ideas. At the same time, due to the lack of in-depth thinking about the details of the project, errors or problems can't be found to solve the problem, so the adoption rate of the design works is low.After the reform, because it is the student is the independent project, the design project is built, so throughout the process, the student's initiative and the exploration are greatly played.It enhances the students' control over the whole design process, so that the passing rate of the design works is greatly improved.

The second increases the interactivity of teachers and students.Due to the relatively weak basis of students, we adopted a one-to-many teaching method, and the teacher was added to the design process.After the students have the design idea, the design details will encounter a lot of difficulties, at this time, it can be more interactive with teachers. Teachers help solve problems and provide solutions.

Thirdly, teachers' business ability is improved. As the technology of Internet of things is the most cutting-edge technology, teachers need to constantly learn new technologies and combine their own electronic technology capabilities to improve their own business capabilities.

\section{Conclusion}

Teaching practice shows that it is feasible to introduce iot technology into electronic information experiment and practical courses.Is driven with iot design project, the teaching method can make students grasp basic knowledge and improve the good operation ability, can form of students' autonomous learning, cooperative learning, exploratory learning, form the learning atmosphere of openness, greatly improved the teaching quality.Meanwhile, the old teaching content is updated to enable students to master the latest and most cutting-edge application technology.Enable students to adapt to the current social and market demands for practical ability.

\section{Acknowledgement}

In this paper, the research was sponsored by school-level Fund Project of Hubei University of Science and Technology (Project No. 2013XB012).

\section{References}

[1] XU Yan-bin,YU Ning,Application of internet of things technology in intelligent campus[J].Hebei Journal of Industrial Science and Technology,2012-06

[2] WANG Li, SU Bo, WANG Fuzhong. Reform on experiment teaching of single-chip microcomputer and cultivation of students' practical ability[C]. 2010 International Conference 
on E-Health Networking, Digital Ecosystems and Technologies, 2010, pp.263-266.

[3] Tian Shisheng, Fu Gangshan. Blended Learning Apreliminary study [J] . E-education Research, 2004, (7):9.

[4] Heikkinen, S. effects of small-group teaching in an electrical engineering course. Global J. of Engng. Educ, 2013,15(3), p.p.171-176.

[5] Tongcheng Huang, Xu Duan, Shiming Jiang, liping Yin. Research on teaching reform of a provincial course: foundation and Application of SCM[J]. World Transactions on Engineering and Technology Education, 2015,13(1), 110-115. 